$7^{\text {th }}$ Jagna International Workshop (2014)

International Journal of Modern Physics: Conference Series

Vol. 36 (2015) 1560007 (16 pages)

(C) The Author

DOI: 10.1142/S2010194515600071

\title{
Weak ergodicity breaking and ageing in anomalous diffusion
}

\author{
Ralf Metzler \\ Institute for Physics \& Astronomy, University of Potsdam, \\ D-14476 Potsdam-Golm, Germany 8 Department of Physics, \\ Tampere University of Technology, FI-33101 Tampere, Finland
}

Published 2 January 2015

\begin{abstract}
Modern single particle tracking techniques and many large scale simulations produce time series $\mathbf{r}(t)$ of the position of a tracer particle. Standardly these are evaluated in terms of the time averaged mean squared displacement. For ergodic processes such as Brownian motion, one can interpret the results of such an analysis in terms of the known theories for the corresponding ensemble averaged mean squared displacement, if only the measurement time is sufficiently long. In anomalous diffusion processes, that are widely observed over many orders of magnitude, the equivalence between (long) time and ensemble averages may be broken (weak ergodicity breaking). In such cases the time averages may no longer be interpreted in terms of ensemble theories. Here we collect some recent results on weakly non-ergodic systems with respect to the time averaged mean squared displacement and the inherent irreproducibility of individual measurements. We also address the phenomenon of ageing, the dependence of physical observables on the time span between initial preparation of the system and the start of the measurement.
\end{abstract}

Keywords: Anomalous diffusion; ensemble average; time average; ageing; nonstationarity.

\section{Introduction}

Following the three groundbreaking papers on the theory of Brownian motion ${ }^{1}$ by Albert Einstein, ${ }^{2}$ Marian Smoluchowski, ${ }^{3}$ and Paul Langevin, ${ }^{4}$ in 1908 Jean Perrin reported the first systematic single particle tracking results in his seminal paper on diffusion. Perrin used microscopic diffusion measurements of small putty particles to determine Avogadro's number via the Einstein-Stokes-Smoluchowski relation. ${ }^{5}$ Due to the relatively short trajectories, Perrin used the ensemble information of many measured, not completely identical particles in his analysis. ${ }^{5}$ Only six years after Perrin's first publication and exactly hundred years ago, in 1914 Ivar Nordlund conceived an experimental setup, that allowed him to record long time traces of

This is an Open Access article published by World Scientific Publishing Company. It is distributed under the terms of the Creative Commons Attribution 3.0 (CC-BY) License. Further distribution of this work is permitted, provided the original work is properly cited. 


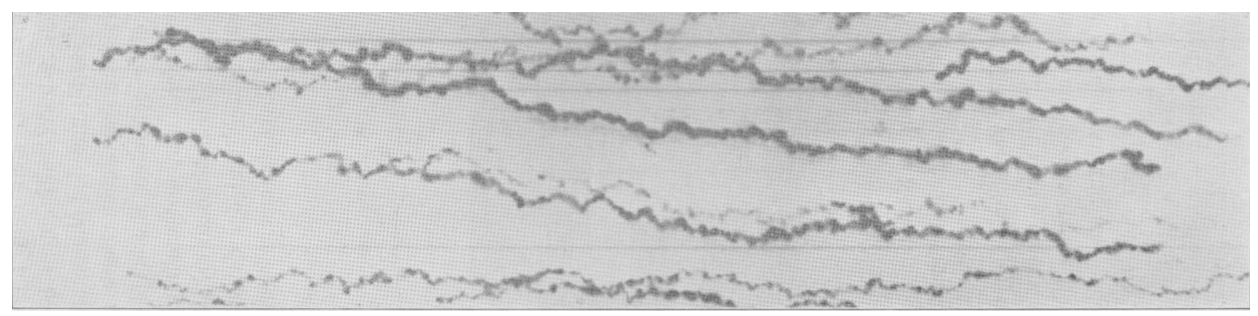

Fig. 1. Sample trajectories of sedimenting mercury droplets measured by Ivar Nordlund in 1914 with his moving film technique, time increases to the right. ${ }^{6}$ The jiggly motion of the droplets superimposed onto the deterministic sedimentation shows the Brownian motion of the droplets.

small mercury droplets on a moving film. From the records he then evaluated single particle trajectories in terms of time averages of the mean squared displacement. ${ }^{6}$ Figure 1 shows typical trajectories measured by Nordlund. ${ }^{6}$ His method was continuously refined in the following two decades, culminating in the measurements of Eugen Kappler, ${ }^{7}$ whose result for Avogadro's number is within $1 \%$ of the current best known value.

Single particle tracking has become a routine tool in living biological cells as well as complex fluids in vitro. ${ }^{8}$ Common tracer particles include fluorescently labelled molecules such as messenger RNA in the cytoplasm of cells or protein channels in their membranes. Without labels, submicron tracers such as endogenous granules or internalised particles such as viruses or plastic spheres can be directly monitored in microscopes.

Consider first a passive tracer particle in a simple liquid such as water. Single particle tracking of this tracer will reproduce the laws of Brownian motion. The ensemble averaged mean squared displacement (MSD)

$$
\left\langle\mathbf{r}^{2}(t)\right\rangle=\int \mathbf{r}^{2} P(\mathbf{r}, t) d \mathbf{r}
$$

obtained as average of $\mathbf{r}^{2}$ over the probability density function $P(\mathbf{r}, t)$ will yield the linear scaling $\left\langle\mathbf{r}^{2}(t)\right\rangle \simeq K_{1} t$ with time $t$, where $K_{1}$ is the diffusion constant. The proportionality factor depends on the spatial dimension. Single particle tracking experiments produce the time series $\mathbf{r}(t)$ of the particle position. Typically, few but long trajectories $\mathbf{r}(t)$ are measured and analysed in terms of the time averaged MSD

$$
\overline{\delta^{2}(\Delta)}=\frac{1}{T-\Delta} \int_{0}^{T-\Delta}[\mathbf{r}(t+\Delta)-\mathbf{r}(t)]^{2} d t
$$

This moving average sums the particle displacements within the lag time $\Delta$ over the time series $\mathbf{r}(t)$ of length (measurement time) $T$. For normal Brownian motion, the long time limit yields ${ }^{9}$

$$
\overline{\delta^{2}(\Delta)} \simeq K_{1} \Delta,
$$

and we find the equivalence $\left\langle\mathbf{r}^{2}(\Delta)\right\rangle=\overline{\delta^{2}(\Delta)}$ of ensemble and time averaged MSDs. This is a restatement of Boltzmann's ergodic hypothesis: long time and ensemble 
averages of physical observables are equivalent. In the following we will also consider the average over individual trajectories,

$$
\left\langle\overline{\delta^{2}(\Delta)}\right\rangle=\frac{1}{N} \sum_{i=1}^{N} \overline{\delta_{i}^{2}(\Delta)}=\frac{1}{T-\Delta} \int_{0}^{T-\Delta}\left\langle[\mathbf{r}(t+\Delta)-\mathbf{r}(t)]^{2}\right\rangle d t
$$

In many systems deviations from Brownian motion are observed. This anomalous diffusion is typically of the power-law form ${ }^{10}$

$$
\left\langle\mathbf{r}^{2}(t)\right\rangle \simeq K_{\alpha} t^{\alpha}
$$

with the anomalous diffusion exponent $\alpha$ and the generalised diffusion coefficient $K_{\alpha}$ of physical dimension $\left[K_{\alpha}\right]=\mathrm{cm}^{2} / \mathrm{sec}^{\alpha}$. We distinguish subdiffusion $(0<\alpha<1)$ and superdiffusion $(\alpha>1)$. Anomalous diffusion is often measured in crowded media, in particular, in living biological cells. ${ }^{11-14}$

Anomalous diffusion loses the universality of Brownian motion, and the MSD (5) is no longer sufficient to uniquely identify a stochastic process. Many different stochastic processes give rise to anomalous diffusion, and they exhibit many different features. The question we address here is the violation of ergodicity: we analyse which processes give rise to the disparity $\left\langle\mathbf{r}^{2}(\Delta)\right\rangle \neq \overline{\delta^{2}(\Delta)}$ and related properties. As we will see, several commonly used anomalous stochastic processes violate ergodicity and effect the irreproducibility of single particle tracking measurements.

\section{Fractional Brownian and Langevin Equation Motion}

The well known Langevin equation in the overdamped limit ${ }^{\mathrm{a}}$

$$
\frac{d x(t)}{d t}=\sqrt{2 K_{1}} \times \xi(t)
$$

is driven by white Gaussian noise of zero mean and correlator $\left\langle\xi(t) \xi\left(t^{\prime}\right)\right\rangle \sim \delta(t-$ $\left.t^{\prime}\right) .{ }^{4,15}$ In contrast to the $\delta$-correlation fractional Gaussian noise (fGn) has the power-law correlation

$$
\left\langle\xi(t) \xi\left(t^{\prime}\right)\right\rangle \sim \alpha K_{\alpha}(\alpha-1)\left|t-t^{\prime}\right|^{\alpha-2}
$$

with exponent $0<\alpha<2$. FGn is known to characterise the tracer motion in viscoelastic environments. ${ }^{16-21}$ Such correlated noise also governs the motion of individual lipids in lipid membranes, ${ }^{22-24}$ and fGn occurs for the motion of a tracer particle in a single file of colloidal particles with excluded volume interactions. ${ }^{25}$ In the case $0<\alpha<1$ the noise-noise correlator has a negative sign, a situation often termed antipersistent noise. In the case $1<\alpha<2$ we speak of persistence.

${ }^{a}$ For simplicity, we will use the one-dimensional notation for the remainder of this chapter. 


\subsection{Fractional Brownian motion (FBM)}

Fractional Brownian motion simply substitutes the white Gaussian noise in the Langevin equation (6) with fGn (7). ${ }^{26,27}$ From a physical point of view, fGn is to be considered an external noise. The resulting ensemble average for the MSD is given by Eq. (5). FBM is ergodic in the sense that the time averaged MSD for unconfined motion becomes ${ }^{28}$

$$
\overline{\delta^{2}(\Delta)} \sim 2 K_{\alpha} \Delta^{\alpha}
$$

in the limit of long $T$. We emphasise that the equality $\overline{\delta^{2}(\Delta)}=\left\langle x^{2}(\Delta)\right\rangle$ indeed holds for a single trajectory in the long measurement time $T$ limit, ${ }^{16}$ as expected for an ergodic process. The approach to ergodicity occurs as a power-law, similar to regular Brownian motion. ${ }^{28}$

In addition to the ergodic behaviour, individual trajectories of FBM are reproducible. More precisely, the amplitude variation of the time averaged $\operatorname{MSD} \overline{\delta^{2}(\Delta)}$ from different realisations of length $T$ around the mean $\left\langle\overline{\delta^{2}(\Delta)}\right\rangle$ is Gaussian. At a fixed lag time $\Delta$, the width of this distribution decreases with increasing measurement time $T,{ }^{29}$ and sufficiently long individual trajectories are therefore in that sense reproducible.

\subsection{Fractional Langevin equation motion}

When we require that the fGn is internal and should fulfil the Kubo generalised fluctuation-dissipation theorem, the resulting particle motion in the overdamped limit is described by the fractional Langevin equation (FLE) ${ }^{30}$

$$
\gamma \int_{0}^{t}\left(t-t^{\prime}\right)^{\alpha-2} \frac{d x\left(t^{\prime}\right)}{d t^{\prime}} d t^{\prime}=\sqrt{\frac{\gamma k_{B} \mathscr{T}}{\alpha(\alpha-1) K_{\alpha}}} \times \xi(t),
$$

for $1<\alpha<2$. Here $k_{B} \mathscr{T}$ represents the thermal energy. In this formulation the long-range correlations of the noise are matched by the memory integral over the friction kernel. In terms of the fractional Caputo derivative ${ }^{31}$

$$
\frac{d^{2-\alpha} x(t)}{d t^{2-\alpha}}=\frac{1}{\Gamma(\alpha-1)} \int_{0}^{t}\left(t-t^{\prime}\right)^{\alpha-2} \frac{d x\left(t^{\prime}\right)}{d t^{\prime}} d t^{\prime} .
$$

Eq. (9) can be rewritten in the compact form

$$
\frac{d^{2-\alpha} x(t)}{d t^{2-\alpha}}=\frac{1}{\Gamma(\alpha-1)} \sqrt{\frac{k_{B} \mathscr{T}}{\gamma \alpha(\alpha-1) K_{\alpha}}} \times \xi(t),
$$

hence the name fractional Langevin equation. ${ }^{32}$ FLE motion is ergodic,

$$
\overline{\delta^{2}(\Delta, T)} \sim\left\langle x^{2}(\Delta) \simeq 2 K_{2-\alpha} \Delta^{2-\alpha} .\right.
$$

Due to the restriction $1<\alpha<2$, FLE motion is therefore subdiffusive. As for FBM, the approach to ergodicity is algebraic. ${ }^{28}$ We note that FLE motion was also used recently in models of active transport in living cells. ${ }^{33}$ 


\subsection{Transient non-ergodicity of FBM E FLE motion}

The MSD for both FBM and FLE motion crosses over to a plateau in confinement, for instance, in case of diffusion in an harmonic potential $V(x)=k x^{2} / 2 .^{34}$ In the case of FBM, no temperature is defined, and the value of the plateau is a function of the anomalous diffusion exponent $\alpha,\left\langle x^{2}\right\rangle_{\mathrm{st}}=K_{\alpha} \Gamma(1+\alpha) / k^{\alpha}$. ${ }^{35}$ The associated time averaged MSD becomes $\left\langle\bar{\delta}_{\text {stat }}\right\rangle=2\left\langle x^{2}\right\rangle_{\text {st }}$. Here the factor two between the MSD and the time averaged MSD is due to the definition (2), which involves twice the stationary value $\left\langle x^{2}\right\rangle_{\text {st }} .{ }^{36}$ In contrast to FBM, FLE motion fulfils the fluctuation-dissipation relation, and the MSD relaxes to the unique plateau value $\left\langle x^{2}\right\rangle_{\text {th }}=k_{B} \mathscr{T} / k$, while the time average converges to $\left\langle\overline{\delta^{2}}{ }_{\text {th }}\right\rangle=2\left\langle x^{2}\right\rangle_{\text {th }} \cdot{ }^{36}$

While for the free FBM and FLE motion ergodic behaviour is found the crossover to the stationary plateau turns out to be transiently non-ergodic. For both FBM and FLE motion the relaxation of the ensemble averaged MSD is exponential. However, for the time averaged MSD the approach is algebraic. For FBM we find ${ }^{36}$

$$
\overline{\delta^{2}(\Delta)} \sim 2\left\langle x^{2}\right\rangle_{\mathrm{st}}-\frac{K_{\alpha} \Gamma(\alpha+1)}{k^{2}} e^{-k \Delta}-\frac{2 \alpha(\alpha-1) K_{\alpha}}{k^{2} \Delta^{2-\alpha}},
$$

and for FLE motion ${ }^{36}$

$$
\overline{\delta^{2}(\Delta)} \sim 2\left\langle x^{2}\right\rangle_{\text {th }}\left(1-\frac{\gamma}{k \Delta^{2-\alpha}}\right) .
$$

This transient weak ergodicity breaking may lead to the false assumption that in the analysis of data the process has not yet relaxed, while the corresponding MSD $\left\langle x^{2}(t)\right\rangle$ already reached the plateau. This algebraic return to the ergodic behaviour represented by the plateau reminds of the algebraic approach to ergodicity of the free motion mentioned above. For single particle tracking experiments of submicron tracer beads in a worm-like micellar solution, this behaviour is indeed shown in Fig. 2. In this example the confinement is exerted by the optical tweezers used to track the particle. ${ }^{19}$

\subsection{Transient ageing of FBM \& FLE motion}

What happens when the system is initially prepared at time $t=0$ and we start the measurement at some later time $t_{a}>0$, the ageing time? We then define the time averaged MSD as ${ }^{37,38}$

$$
\overline{\delta^{2}(\Delta)}=\frac{1}{T-\Delta} \int_{t_{a}}^{t_{a}+T-\Delta}[x(t+\Delta)-x(t)]^{2} d t .
$$

A Brownian system naturally shows no dependence on $t_{a}$. Even though the process is asymptotically ergodic, however, we observe a transient dependence on $t_{a}$ for processes driven by fGn. In general, for these processes it is found that the time average MSD always contains the two additive terms, ${ }^{37}$

$$
\left\langle\overline{\delta^{2}(\Delta)}\right\rangle=f_{\mathrm{st}}(\Delta)+f_{\text {age }}\left(\Delta ; t_{a}, T\right) .
$$

The stationary term depends only on $\Delta$, while the second, ageing term explicitly depends on $T$ and $t_{a}$. 


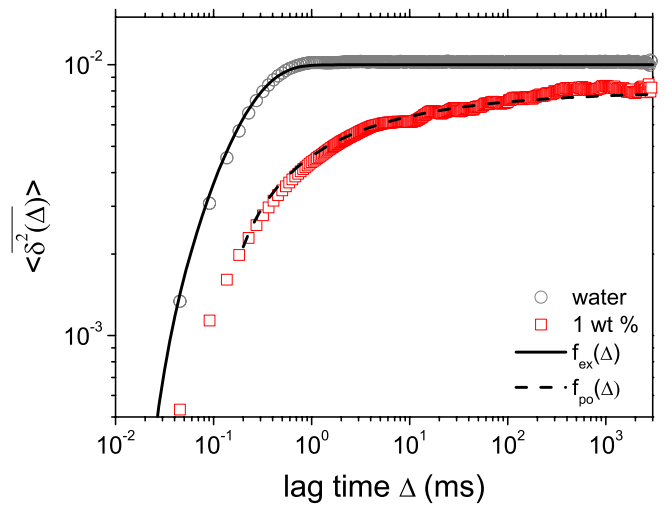

Fig. 2. The time averaged MSD of submicron tracer beads in water (circles) and a viscoelastic solution with $1 \%$ worm-like micelles (squares). ${ }^{19}$ The measurement is based on optical tweezers tracking, so that the initial free motion of the tracer bead eventually becomes confined by the tweezers potential. The time averaged MSD in water relaxes exponentially (full line), while in the worm-like micellar solution we observe the algebraic relaxation of Eq. (14), shown by the dashed line.

Free FLE motion has a stationary term featuring subdiffusion, $f_{\mathrm{st}} \simeq \Delta^{2-\alpha}$, and the ageing term decays as $f_{\text {age }} \simeq 1 / T$ as long as the initial velocity distribution is not thermal. In the limit $t_{a} \gg T$, we find the ageing time dependence ${ }^{37}$

$$
f_{\text {age }} \simeq t_{a}^{-2 \alpha} \text {. }
$$

Under confinement FLE motion the term $f_{\text {st }}$ has a power-law approach to the thermal plateau value, while again $f_{\text {age }} \simeq 1 / T$. Interestingly, a different $t_{a}$-scaling is followed by the ageing term, ${ }^{37}$

$$
f_{\text {age }} \simeq t_{a}^{2 \alpha-6} .
$$

Confined FBM has $f_{\text {age }} \simeq 1 / T$, however, the ageing term shows the exponential decay $^{37}$

$$
f_{\text {age }} \sim x_{0}^{2} \exp \left(-2 k t_{a}\right)
$$

\section{Subdiffusive Continuous Time Random Walks}

As discussed in the previous section, FBM and FLE motion reach the ergodic behaviour algebraically, similar to Brownian motion. For sufficiently long measurements, individual trajectories become fully reproducible, and ergodicity is achieved in every single trajectory. Here we introduce a process, for which ergodicity is broken asymptotically, and even for long measurement times $T$ individual trajectories never become reproducible. This process is the well-known Scher-Montroll-Weiss continuous time random walk (CTRW): ${ }^{39-41}$ after each jump a random walker is trapped (immobilised) for some waiting time $t$ before it is allowed to jump again. The waiting times $t$ are independent random variables, that is, CTRWs are renewal 
processes. Waiting times are distributed identically with the waiting time probability density function $\psi(t)$. The form proposed originally by Scher and Montroll is the power-law ${ }^{40}$

$$
\psi(t) \simeq \frac{\tau^{\alpha}}{t^{1+\alpha}}, \quad 0<\alpha<1
$$

With this distribution of waiting times, the process leads to the subdiffusive MSD (5). ${ }^{40,41}$ Due to the range of $\alpha$, no characteristic waiting time $\langle t\rangle=\int_{0}^{\infty} t \psi(t) d t \rightarrow \infty$ exists. This scale-free nature of the CTRW process no longer possesses a time scale that allows one to distinguish a single or few jumps from many jumps. Typically, in a given trajectory longer and longer individual waiting events occur which can become of the order of the measurement time $T$, no matter how long we run the measurement.

CTRW-type stochastic motion was observed in a wide range of systems, spanning the motion of charge carriers in amorphous semiconductors, ${ }^{40}$ the dispersion of tracer chemicals in subsurface aquifers, ${ }^{42}$ as well as the motion of tracer beads in cross-linked semiflexible actin gels ${ }^{43}$ and of functionalised colloidal particles facing complementarily functionalised surfaces. ${ }^{44}$ In living cells, the motion of lipid and insulin granules in the cell cytoplasm ${ }^{17,18}$ as well as of protein channels in the plasma membrane ${ }^{45}$ follow the law (20).

The lack of a characteristic waiting time scale effects weak ergodicity breaking, ${ }^{46,47}$ and the time averaged MSD becomes ${ }^{48,49}$

$$
\left\langle\overline{\delta^{2}(\Delta)}\right\rangle \sim 2 \frac{K_{\alpha}}{\Gamma(1+\alpha)} \frac{\Delta}{T^{1-\alpha}}, \quad \Delta \ll T,
$$

which shows a clear disparity with the ensemble averaged MSD (5). Despite the anomalous nature of the process, the dependence of the time averaged MSD (21) on the lag time $\Delta$ is the same as for Brownian motion. Only the fact that the amplitude decays as function of the measurement time $T$ reflects the anomaly: while the process evolves in time, increasingly longer individual waiting times occur and cause a decay of the effective diffusivity $\simeq K_{\alpha} / T^{1-\alpha}$. This behaviour also leads to severe changes in the interaction of a particle with a reactive surface ${ }^{50,51}$ and the exploration of phase scape. ${ }^{52}$

Figure 3 shows the time averaged MSD for individual realisations of a subdiffusive CTRW with $\alpha=0.5$. We notice a distinct scatter of the amplitudes between the realisations. Moreover, while for most realisations the predicted linear slope $\left\langle\overline{\delta^{2}(\Delta)}\right\rangle \simeq \Delta$ is observed, some of the time traces also show variations in the local slope. Such amplitude scatter and local slope variations are a common feature in many experiments, compare Refs. $17,18,45,53$. We can quantify the amplitude scatter in terms of the dimensionless ratio $\xi=\overline{\delta^{2}(\Delta)} /\left\langle\overline{\delta^{2}(\Delta)}\right\rangle$. The corresponding distribution of relative amplitudes, $\phi_{\alpha}(\xi)$ in the case of the subdiffusive CTRW becomes a one-sided Lévy stable distribution. ${ }^{9,48}$ This distribution for the limit 


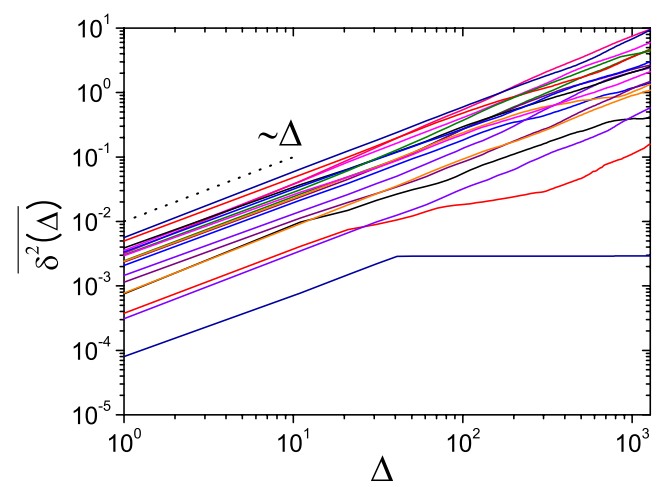

Fig. 3. (Color online) Individual trajectories of a scale-free, subdiffusive CTRW with $\alpha=0.5$ exhibit the linear lag time dependence predicted by Eq. (21), with smaller local variations of the slope. In addition, there is a clear scatter of the amplitudes between individual trajectories. These features reflect the influence of individual long waiting time events.

$T \rightarrow \infty$ demonstrates that no matter how long we average the motion of the particle, on the single trajectory level the time averaged MSD of this process always remains a random quantity. In the special case $\alpha=1 / 2$ we find the Gaussian form

$$
\phi_{1 / 2}(\xi)=\frac{2}{\pi} \exp \left(-\frac{\xi^{2}}{\pi}\right) \text {. }
$$

Its maximum is at $\xi=0$, reflecting completely stalled trajectories during the measurement time $T$. Mobile trajectories with $\xi>0$ are distributed as a half Gaussian. When $\alpha$ increases towards the Brownian value $\alpha=1$, a peak emerges at $\xi=1$. In the Brownian case $\alpha=1$, ergodicity is restored, and $\phi_{1}(\xi)=\delta(\xi-1)$ indicates that for sufficiently long trajectories each realisation is fully reproducible. This behaviour in terms of $\phi(\xi)$ is independent of an external potential, ${ }^{54,55}$ due to the fact that the ratio $\overline{\delta^{2}(\Delta)} /\left\langle\overline{\delta^{2}(\Delta)}\right\rangle$ is equal to the ratio $n(T) /\langle n(T)\rangle$ of the number of jumps. ${ }^{38}$

Under confinement, for instance, by an harmonic external potential within a finite domain with reflecting walls, the time averaged MSD of subdiffusive CTRWs does not converge to the thermal plateau of the ensemble averaged MSD. Instead, the time averaged MSD scales like ${ }^{55,56}$

$$
\left\langle\overline{\delta^{2}(\Delta)}\right\rangle \sim\left(\left\langle x^{2}\right\rangle_{B}-\langle x\rangle_{B}^{2}\right) \frac{2 \sin (\pi \alpha)}{(1-\alpha) \pi \alpha}\left(\frac{\Delta}{T}\right)^{1-\alpha}
$$

for $\Delta \ll T$ and $\Delta \gg\left(1 /\left[K_{\alpha} \lambda_{1}\right]\right)^{1 / \alpha} . \lambda_{1}$ represents the lowest non-zero eigenvalue of the Fokker-Planck operator in the confining potential, a measure for the time scale when the particle engages with the confinement. The result (23) is universal in so far as only the prefactor depends on the very form of the confining potential $V(x)$. It involves the first and second moments of the Boltzmann distribution, $\left\langle x^{j}\right\rangle_{B}=\int x^{j} \exp \left(-V(x) /\left[k_{B} \mathscr{T}\right]\right) d x / \mathscr{Z}$. The normalisation factor is the partition $\mathscr{Z}=\int \exp \left(-V(x) /\left[k_{B} \mathscr{T}\right]\right) d x$. The analysis shows that in this scale free process weak non-ergodicity remains present even in the limit of long measurements. 


\subsection{Ageing behaviour of subdiffusive CTRW processes}

CTRW processes with diverging time scale display ageing effects. ${ }^{57,58}$ We already saw ageing in the presence of the measurement time $T$ in the time averaged MSD (21). Ageing is due to the non-stationarity of the process. Thus, in subdiffusive CTRWs the two-point correlation $\left\langle x\left(t_{1}\right) x\left(t_{2}\right)\right\rangle=f\left(t_{1} / t_{2}\right)$ is not a function of the difference $\left|t_{2}-t_{1}\right|$ of the two times but their ratio. ${ }^{55}$ This breakdown of stationarity removes the time translation invariance of stationary processes and needs to be taken into consideration in experiments, in which the start of the recording of the trajectories occurs only at some (ageing) time $t_{a}>0$ after the original initialisation of the system dynamics at $t=0$.

For the regular MSD, for sufficiently long ageing times $t_{a}$ this leads to a crossover from the scaling $\left\langle x^{2}(t)\right\rangle \simeq K_{\alpha} t / t_{a}^{1-\alpha}$ in the ageing-dominated regime $t \ll t_{a}$ to the scaling (5) when the system evolves for much longer than the ageing time, $t \gg t_{a} \cdot{ }^{38,58}$ In the same situation the time averaged MSD (15) behaves much simpler and features the multiplicative, universal correction factor ${ }^{38}$

$$
\Lambda_{\alpha}\left(t_{a} / T\right)=\left(1+\frac{t_{a}}{T}\right)^{\alpha}-\left(\frac{t_{a}}{T}\right)^{\alpha} .
$$

This factor solely depends on the ratio $t_{a} / T$ of ageing time $t_{a}$ and measurement time $T$. Thus, apart from the amplitude, the scaling of the time averaged MSD (15) as function of the lag time $\Delta$ remains unaffected, an important piece of knowledge when the exact age $t_{a}$ of the process is not precisely known. ${ }^{38}$

Ageing of a subdiffusive CTRW process gives rise to another remarkable feature. Namely, the probability to observe at least one jump in an aged trajectory of length $T$ decreases algebraically with the ageing time $t_{a} \cdot{ }^{38}$ This property of the population splitting of particles into a mobile and a fully immobile fraction has to be taken into account when we want to deduce the anomalous diffusion constant from aged trajectories. ${ }^{38}$ We note that also the first passage time behaviour of aged CTRW processes exhibits an explicit dependence on the ageing time $t_{a}$. In particular, interesting crossovers between different scaling regimes occur, a fact that may be used to deduce the age $t_{a}$ of a system from sufficiently long first passage data. ${ }^{59}$

More specifically, in an aged system the start of the measurement at $t_{a}$ typically finds the system during one of the long waiting time events. It can be shown that the occurrence of the first jump event in this case at the so-called forward waiting time $t_{1}$ is distributed according to, ${ }^{60-62}$

$$
\psi_{1}\left(t_{1} \mid t_{a}\right)=\frac{\sin (\pi \alpha)}{\pi} \frac{t_{a}^{\alpha}}{t_{1}^{\alpha}\left(t_{a}+t_{1}\right)} .
$$

At long waiting times $t_{a} \gg t_{1}$ the distribution of the forward waiting time is thus broader than the regular waiting times $t$ in $\psi(t)$. In an aged CTRW all subsequent jumps then follow the law $\psi(t)$ again. Still, due to the macroscopic memory inherent in CTRW processes, ${ }^{10}$ the influence of the ageing time persists until the evolution is much longer than $t_{a}$. In a modified CTRW model, in which every jump is dominated 
by the forward waiting time (25), the dynamics of the process is significantly slowed down, giving rise to logarithmic time evolutions. ${ }^{63}$ These can be connected to single file systems in which each particle separately becomes trapped with a scale-free distribution of trapping times $\psi(t) .{ }^{64}$

\section{Correlated Continuous Time Random Walks}

What if we do away with the renewal property of the previously discussed CTRW process? One way to include correlations into the CTRW process is to consider a model when successive waiting times are only separated by an incremental change. Physically, this could reflect the motion in a quenched environment, in which locally the motion is dominated by a given mobility with small variations. We could thus imagine that the current waiting $\tau_{i}$ is composed of increments in the form ${ }^{65-67}$

$$
\tau_{i}=\left|\xi_{1}+\xi_{2}+\ldots+\xi_{i-1}\right| \text {. }
$$

If the $\xi_{i}$ are distributed according to a Lévy stable law defined in terms of its Fourier transform $\exp \left(-c_{\gamma}|k|^{\gamma}\right)$ with $0<\gamma<2$, then the process leads to anomalous diffusion governed by Eq. (5) with the anomalous diffusion exponent $\alpha=\gamma /(1+\gamma)$. Its range is $0<\alpha<2 / 3 .{ }^{65,66}$ This model features a stretched exponential mode relaxation $P(k, t) \simeq \exp \left(-c t^{1 / 2}\right)$ in the limit $\gamma=2$, while for for $0<\gamma<2$ a power-law form $P(k, t) \simeq t^{-\gamma}$ is obtained. ${ }^{67}$ There also exist alternative models to correlated jump processes, see the discussion in Refs. 68 and 69 and the citations therein.

The absolute value in the law (26) implies that the mean waiting time keeps growing with $T$ and diverges in the long time limit. The time averaged MSD ${ }^{67}$

$$
\left\langle\overline{\delta^{2}(\Delta)}\right\rangle \simeq \frac{\Delta}{T^{1-\gamma /(1+\gamma)}}
$$

shows the weakly non-ergodic behaviour of the correlated CTRW process. It also features ageing effects demonstrated by the temporal decay of the response of the system to a periodic driving force. ${ }^{67}$ Individual trajectories show a pronounced amplitude scatter. ${ }^{65}$

A similar trick can be used to correlate subsequent jump lengths. The MSD of this process is then given exactly by ${ }^{65}$

$$
\left\langle x(t)^{2}\right\rangle \simeq \frac{t(t+1)(2 t+1) \sigma^{2}}{4},
$$

for a Gaussian distribution of jump increments with variance $\sigma^{2}$. This process thus has the cubic long time scaling behaviour $\left\langle x(t)^{2}\right\rangle \simeq t^{3}$. The associated time averaged MSD scales quadratically, ${ }^{65}$

$$
\left\langle\overline{\delta^{2}(\Delta)}\right\rangle \simeq \Delta^{2} T
$$

for $\Delta \ll T$. Thus, also this process is weakly non-ergodic. ${ }^{65}$ 


\section{Heterogeneous Diffusion Processes}

Let us now address a seemingly much simpler scenario, namely, a diffusion process with a space-dependent diffusivity $K(x)$. Such descriptions were used to model turbulence $^{70}$ or diffusion in heterogenous porous media. ${ }^{71,72}$ In biological cells, local variations of the diffusion coefficient were indeed recently mapped out. ${ }^{73} \mathrm{We}$ consider the Langevin equation ${ }^{74}$

$$
\frac{d x(t)}{d t}=\sqrt{2 K(x)} \times \xi(t)
$$

where the multiplicative noise $\xi(t)$ is white and Gaussian with zero mean. Using the Stratonovich interpretation this heterogeneous diffusion process (HDP) can be shown to be weakly non-ergodic.

Consider the power-law form $K(x) \simeq K_{0}|x|^{\beta}$ for the diffusivity. The MSD is then given by ${ }^{74}$

$$
\left\langle x^{2}(t)\right\rangle=\frac{\Gamma(p+1 / 2)}{\pi^{1 / 2}}\left(\frac{2}{p}\right)^{2 p}\left(K_{0} t\right)^{p},
$$

with the exponent $p=2 /(2-\beta)$. For $\beta<0$ this process is therefore subdiffusive, while for $0<\beta<2$ it is superdiffusive. ${ }^{74}$ The time averaged MSD in the limit $\Delta \ll T$ exhibits the linear dependence ${ }^{74}$

$$
\left\langle\overline{\delta^{2}(\Delta)}\right\rangle=\frac{\Gamma(p+1 / 2)}{\pi^{1 / 2}}\left(\frac{2}{p}\right)^{2 p} K_{0}^{p} \frac{\Delta}{T^{1-p}}
$$

on the lag time, valid for both sub- and superdiffusive regimes. This implies the exact connection $\left\langle\overline{\delta^{2}(\Delta)}\right\rangle=(\Delta / T)^{1-p}\left\langle x^{2}(\Delta)\right\rangle$ with the ensemble averaged MSD.

Interestingly, despite the simplicity of the HDP process we again observe a weakly non-ergodic behaviour. Similar results follow in the case of fast (exponential) and slow (logarithmic) variations of the diffusivity $K(x)$ with the particle position $x .{ }^{75}$ We note that for the exponential case the square root scaling $\left\langle\overline{\delta^{2}(\Delta)}\right\rangle \simeq \Delta^{1 / 2}$ was observed. ${ }^{75}$ In the context of imaged diffusion in cells the HDP process with power-law $x$-dependence of $K(x)$ was also generalised to two dimensions. ${ }^{76}$

\section{Scaled Brownian Motion}

What if we consider a time-dependent diffusion coefficient instead of the $x$ dependence? As pointed out by Fuliński already, ${ }^{77}$ such experimentally observed variations of the diffusivity ${ }^{78}$ may cause weakly non-ergodic behaviour in analogy to the spatial dependence in the HDP process above. For a power-law time dependence of the diffusivity this process is so-called scaled Brownian motion (SBM). ${ }^{79}$ Let us start with the Langevin equation

$$
\frac{d x(t)}{d t}=\sqrt{2 \mathscr{K}(t)} \times \xi(t),
$$


where $\xi(t)$ is white Gaussian noise with zero mean. The diffusion coefficient is given by

$$
\mathscr{K}(t)=\alpha K_{\alpha} t^{\alpha-1}
$$

where $0<\alpha<2$. This process obviously leads to the MSD (5). Concurrently, the time averaged MSD has the exact form ${ }^{80}$

$$
\left\langle\overline{\delta^{2}(\Delta)}\right\rangle=\frac{2 K_{\alpha} t^{1+\alpha}}{(\alpha+1)} \frac{\left[1-(\Delta / T)^{1+\alpha}-(1-\Delta / T)^{1+\alpha}\right]}{T-\Delta} .
$$

For $\Delta \ll T$, the linear $\Delta$-scaling is recovered, ${ }^{81}$

$$
\left\langle\overline{\delta^{2}(\Delta)}\right\rangle \sim 2 K_{\alpha} \frac{\Delta}{T^{1-\alpha}}
$$

in both the sub- and superdiffusive cases. Thus, again we obtain a weakly nonergodic behaviour given by the disparity between ensemble and time averaged MSD. However, different to the above weakly non-ergodic processes, SBM features fully reproducible trajectories in the long time limit. ${ }^{80,81}$ As discussed in Ref. 80 in detail, the time dependent diffusivity $\mathscr{K}(t)$ may appear as a simple and natural choice for the description of anomalous diffusion processes. However, $\mathscr{K}(t)$ actually reflects a time-dependent temperature, ${ }^{77,80}$ and thus leads to unphysical behaviour in thermalised systems, in particular, when the data are from a confined system, for instance, when the trajectories are measured by optical tweezers methods. ${ }^{80}$

\section{Conclusions}

Single particle tracking is increasingly becoming a standard tool to study the motion of tracer particles in systems such as complex fluids or even living biological cells. Concurrently, single particle traces are evaluated in large scale computer simulations, for instance, to detect inhomogeneous motion in a population of simulated particles. To evaluate the garnered time series one typically uses the time averaged MSD. As we showed here, when the motion of the particle is anomalous, care has to be taken to evaluate the results in a physically meaningful way. Due to the occurrence of transient or asymptotic weak ergodicity breaking, one cannot simply compare the results for the time averages with the known behaviour of the corresponding ensemble averages.

Apart from the processes discussed herein, non-ergodic behaviour also occurs in other stochastic processes, including the ultraweakly non-ergodic Lévy walks ${ }^{82-84}$ where the disparity between ensemble and time averaged MSDs only amounts to a constant factor. Diffusion on random, fractal percolation clusters was shown to be ergodic. ${ }^{85}$ We also note that in some systems combinations of stochastic processes have to be applied to capture the observed data. ${ }^{17,18,45,86-88}$ 
The diagnosis of a given data set for the exact underlying stochastic process ${ }^{11-14}$ requires the analysis of several complementary quantities. We mention the amplitude scatter statistics, ${ }^{29}$ increment autocorrelations, ${ }^{9,23}$ higher order moments, ${ }^{89,}, 90$ mean maximal excursion methods, ${ }^{89}$ p-variation,,${ }^{91,92}$ and the analysis of the distribution of the apparent diffusivity. ${ }^{93}$

\section{Acknowledgments}

The author acknowledges funding from the Academy of Finland within the Finland Distinguished Professor scheme.

\section{References}

1. R. Brown, Phil. Mag. 4, 161, 1828.

2. A. Einstein, Ann. d. Physik 17, 549 (1905).

3. M. von Smoluchowsky, Ann. Phys. (Leipzig) 21, 756 (1906).

4. P. Langevin, C. R. Acad. Sci. Paris 146, 530 (1908).

5. J. Perrin, C. R. Acad. Sci. Paris 146, 967 (1908).

6. I. Nordlund, Z. Phys. Chem. 87, 40 (1914).

7. E. Kappler, Ann. d. Phys. (Leipzig) 11, 233 (1931).

8. C. Bräuchle, D. C. Lamb, and J. Michaelis, Single Particle Tracking and Single Molecule Energy Transfer (Wiley-VCH, Weinheim, Germany, 2012); X. S. Xie, P. J. Choi, G.-W. Li, N. K. Lee, and G. Lia, Annu. Rev. Biophys. 37, 417 (2008).

9. S. Burov, J.-H. Jeon, R. Metzler, and E. Barkai, Phys. Chem. Chem. Phys. 13, 1800 (2011).

10. R. Metzler and J. Klafter, Phys. Rep. 339, 1 (2000); J. Phys. A 37, R161 (2004).

11. M. J. Saxton and K. Jacobson, Annu. Rev. Biophys. Biomol. Struct. 26, 373 (1997).

12. E. Barkai, Y. Garini, and R. Metzler, Physics Today 65(8), 29 (2012).

13. F. Höfling and T. Franosch, Rep. Prog. Phys. 76, 046602 (2013).

14. I. M. Sokolov, Soft Matter 8, 9043 (2012).

15. W. T. Coffey and Yu. P. Kalmykov, The Langevin Equation: With Applications to Stochastic Problems in Physics, Chemistry and Electrical Engineering (World Scientific, Singapore, 2012).

16. I. Goychuk, Phys. Rev. E 80, 046125 (2009); Adv. Chem. Phys. 150, 187 (2012).

17. J.-H. Jeon, V. Tejedor, S. Burov, E. Barkai, C. Selhuber-Unkel, K. Berg-Sørensen, L. Oddershede, and R. Metzler, Phys. Rev. Lett. 106, 048103 (2011).

18. S. M. A. Tabei, S. Burov, H. Y. Kim, A. Kuznetsov, T. Huynh, J. Jureller, L. H. Philipson, A. R. Dinner, and N. F. Scherer, Proc. Natl. Acad. Sci. USA 110, 4911 (2013).

19. J.-H. Jeon, N. Leijnse, L. B. Oddershede, and R. Metzler, New J. Phys. 15, 045011 (2013).

20. S. C. Weber, A. J. Spakowitz, and J. A. Theriot, Phys. Rev. Lett. 104, 238102 (2010).

21. J. Szymanski and M. Weiss, Phys. Rev. Lett. 103, 038102 (2009).

22. G. R. Kneller, K. Baczynski, and M. Pasenkiewicz-Gierula, J. Chem. Phys. 135, 141105 (2011).

23. J.-H. Jeon, H. Martinez-Seara Monne, M. Javanainen, and R. Metzler, Phys. Rev. Lett. 109, 188103 (2012).

24. M. Javanainen, H. Hammaren, L. Monticelli, J.-H. Jeon, R. Metzler, and I. Vattulainen, Faraday Disc. 161, 397 (2013). 
25. T. E. Harris, J. Appl. Prob. 2(2), 323 (1965).

26. A. N. Kolmogorov, Dokl. Acad. Sci. USSR 26, 115 (1940).

27. B. B. Mandelbrot and J. W. van Ness, SIAM Rev. 1, 422 (1968).

28. W. Deng and E. Barkai, Phys. Rev. E 79, 011112 (2009).

29. J.-H. Jeon and R. Metzler, J. Phys. A 43, 252001 (2010).

30. R. Zwanzig, Nonequilibrium Statistical Mechanics (Oxford University Press, Oxford, UK, 2001).

31. F. Mainardi, Fractional calculus and waves in linear viscoelasticity (Imperial College Press, London, 2010).

32. E. Lutz, Phys. Rev. E 64, 051106 (2001).

33. I. Goychuk, V. O. Kharchenko, and R. Metzler, PLoS ONE 9, e91700 (2014); Phys. Chem. Chem. Phys. DOI: 10.1039/C4CP01234H.

34. J.-H. Jeon and R. Metzler, Phys. Rev. E 81, 021103 (2010).

35. O. Yu. Sliusarenko, V. Yu. Gonchar, A. V. Chechkin, I. M. Sokolov, and R. Metzler, Phys. Rev. E 81, 041119 (2010).

36. J.-H. Jeon and R. Metzler, Phys. Rev. E 85, 021147 (2012).

37. J. Kursawe, J. H. P. Schulz, and R. Metzler, Phys. Rev. E 88, 062124 (2013).

38. J. H. P. Schulz, E. Barkai, and R. Metzler, Phys. Rev. Lett. 110, 020602 (2013); Phys. Rev. X 4, 011028 (2014).

39. E. W. Montroll and G. H. Weiss, J. Math. Phys. 6, 167 (1965).

40. H. Scher and E. W. Montroll, Phys. Rev. B 12, 2455 (1975).

41. B. D. Hughes, Random Walks and Random Environments, Volume 1: Random Walks (Oxford University Press, Oxford, 1995).

42. H. Scher, G. Margolin, R. Metzler, J. Klafter, and B. Berkowitz, Geophys. Res. Lett. 29, 1061 (2002).

43. I. Y. Wong, M. L. Gardel, D. R. Reichman, E. R. Weeks, M. T. Valentine, A. R. Bausch, and D. A. Weitz, Phys. Rev. Lett. 92, 178101 (2004).

44. Q. Xu, L. Feng, R. Sha, N. C. Seeman, and P. M. Chaikin, Phys. Rev. Lett. 106, $228102(2011)$.

45. A. V. Weigel, B. Simon, M. M. Tamkun, and D. Krapf, Proc. Nat. Acad. Sci. USA 108, 6438 (2011).

46. J.-P. Bouchaud, J. Phys. I 2, 1705 (1992).

47. G. Bel and E. Barkai, Phys. Rev. Lett. 94, 240602 (2005).

48. Y. He, S. Burov, R. Metzler, and E. Barkai, Phys. Rev. Lett. 101, 058101 (2008).

49. A. Lubelski, I. M. Sokolov, and J. Klafter, Phys. Rev. Lett. 100, 250602 (2008).

50. M. A. Lomholt, I. M. Zaid, and R. Metzler, Phys. Rev. Lett. 98, 200603 (2007); I. M. Zaid, M. A. Lomholt, and R. Metzler, Biophys. J. 97, 710 (2009).

51. M. J. Skaug, A. M. Lacasta, L. Ramirez-Piscina, J. M. Sancho, K. Lindenberg, and D. K. Schwartz, Soft Matter 10, 753 (2014); M. Khoury, A. M. Lacasta, J. M. Sancho, and K. Lindenberg, Phys. Rev. Lett. 106, 090602 (2011).

52. G. Bel and E. Barkai, J. Phys. Cond. Mat. 17, S4287 (2005); Phys. Rev. Lett. 94, 240602 (2005); A. Rebenshtok and E. Barkai, J. Stat. Phys. 133, 565 (2008); Phys. Rev. Lett. 99, 210601 (2007).

53. I. Golding and E. C. Cox, Phys. Rev. Lett. 96, 098102 (2006).

54. I. M. Sokolov, E. Heinsalu, P. Hänggi and I. Goychuk, Europhys. Lett. 86, 30009 (2009).

55. S. Burov, R. Metzler, and E. Barkai, Proc. Natl. Acad. Sci. USA 107, 13228 (2010).

56. T. Neusius, I. M. Sokolov, and J. C. Smith, Phys. Rev. E 80, 011109 (2009).

57. C. Monthus and J.-P. Bouchaud, J. Phys. A 29, 3847 (1996).

58. E. Barkai and Y. C. Cheng, J. Chem. Phys. 118, 6167 (2003). 
59. H. Krüsemann, A. Godec, and R. Metzler, Phys. Rev. E 89, 040101(R) (2014).

60. E. B. Dynkin, Izv. Akad. Nauk. SSSR Ser. Math. 19, 247 (1955); Selected Translations Math. Stat. Prob. 1, 171 (1961).

61. C. Godrèche and J. M. Luck, J. Stat. Phys. 104, 489 (2001); E. Barkai and Y.-C. Cheng, J. Chem. Phys. 118, 6167 (2003); E. Barkai, Phys. Rev. Lett. 90, 104101 (2003).

62. T. Koren, M. A. Lomholt, A. V. Chechkin, J. Klafter, and R. Metzler, Phys. Rev. Lett. 99, 160602 (2007).

63. M. A. Lomholt, L. Lizana, R. Metzler, and T. Ambjörnsson, Phys. Rev. Lett. 110, 208301 (2013).

64. L. P. Sanders, M. A. Lomholt, L. Lizana, K. Fogelmark, R. Metzler, and T. Ambjörnsson, E-print arXiv:1311.3790.

65. V. Tejedor and R. Metzler, J. Phys. A 43, 082002 (2010).

66. M. Magdziarz, R. Metzler, W. Szczotka, and P. Zebrowski, Phys. Rev. E 85, 051103 (2012).

67. M. Magdziarz, R. Metzler, W. Szczotka, and P. Zebrowski, J. Stat. Mech. P04010 (2012).

68. A. V. Chechkin, M. Hofmann, and I. M. Sokolov, Phys. Rev. E 80, 031112 (2009).

69. J. H. P. Schulz, A. V. Chechkin, and R. Metzler, J. Phys. A. 46, 475001 (2013).

70. L. F. Richardson, Proc. Roy. Soc. London, Ser. A 110, 709 (1926); A. S. Monin and A. M. Yaglom, Statistical Fluid Mechanics (MIT Press, Cambdridge MA, 1971).

71. M. Dentz, P. Gouze, A. Russian, J. Dweik, and F. Delay, Adv. Water Res. 49, 13 (2012).

72. C. Loverdo et al., Phys. Rev. Lett. 102, 188101 (2009).

73. T. Kühn, T. O. Ihalainen, J. Hyväluoma, N. Dross, S. F. Willman, J. Langowski, M. Vihinen-Ranta, and J. Timonen, PLoS One 6, e22962 (2011).

74. A. G. Cherstvy, A. V. Chechkin, and R. Metzler, New J. Phys. 15, 083039 (2013).

75. A. G. Cherstvy and R. Metzler, Phys. Chem. Chem. Phys. 15, 20220 (2013).

76. A. V. Cherstvy, A. V. Chechkin, and R. Metzler, Soft Matter 10, 1591 (2014).

77. A. Fuliński, Phys. Rev. E 83, 061140 (2011); J. Chem. Phys. 138, 021101 (2013).

78. M. Platani, I. Goldberg, A. I. Lamond, and J. R. Swedlow, Nature Cell Biol. 4, 502 (2002).

79. S. C. Lim and S. V. Muniandy, Phys. Rev. E 66, 021114 (2002).

80. J.-H. Jeon, A. V. Chechkin, and R. Metzler, Phys. Chem. Chem. Phys. DOI: 10.1039/c4cp02019g.

81. F. Thiel and I. M. Sokolov, Phys. Rev. E 89, 012115 (2014).

82. G. Zumofen and J. Klafter, Physica D 69, 436 (1993).

83. A. Godec and R. Metzler, Phys. Rev. Lett. 110, 020603 (2013); Phys. Rev. E 88, 012116 (2013).

84. D. Froemberg and E. Barkai, Phys. Rev. E 87, 030104(R) (2013); Phys. Rev. E 88, 024101 (2013).

85. Y. Meroz, I. Eliazar, and J. Klafter, J. Phys. A 42, 434012 (2009); Y. Meroz, I. M. Sokolov, and J. Klafter, Phys. Rev. Lett. 110, 090601 (2013).

86. T. Akimoto, E. Yamamoto, K. Yasuoka, Y. Hirano, and M. Yasui, Phys. Rev. Lett. 107, 178103 (2011).

87. J.-H. Jeon, E. Barkai, and R. Metzler, J. Chem. Phys. 139, 121916 (2013).

88. S. Eule and R. Friedrich, Phys. Rev. E 87, 032162 (2013).

89. V. Tejedor, O. Bénichou, R. Voituriez, R. Jungmann, F. Simmel, C. Selhuber-Unkel,

L. Oddershede, and R. Metzler, Biophys. J. 98, 1364 (2010).

90. D. Ernst, J. Kohler, and M. Weiss, Phys. Chem. Chem. Phys. 16, 7686 (2014). 
91. M. Magdziarz, A. Weron, K. Burnecki, and J. Klafter, Phys. Rev. Lett. 103, 180602 (2009)

92. K. Burnecki, E. Kepten, J. Janczura, I. Bronshtein, Y. Garini, and A. Weron, Biophys. J. 103, 1839 (2012).

93. M. Bauer, R. Valiullin, G. Radons, and J. Kaerger, J. Chem. Phys. 135, 144118 (2011). 